CASE REPORT

\title{
Ciliary body adenoma of non-pigmented epithelium
}

\section{S Mansoor, A Qureshi}

J Clin Pathol 2004;57:997-998. doi: 10.1136/jcp.2004.017871

\begin{abstract}
Adenomas of the non-pigmented epithelium of the ciliary body are rare neoplasms and most of the studies are in the form of case reports. There are only 27 documented cases of acquired neoplasm of the non-pigmented ciliary epithelium (NPCE) reported in the English literature. In most reports, there was a clinical suspicion of melanoma and the diagnosis of NPCE adenoma was made on histopathological evaluation of the resected tissue. The entity has not been reported in the Pakistani population to date. This report describes a case of ciliary body adenoma of NPCE in a 27 year old Pakistani man. The histological and immunohistochemical profiles were typical of the adenomas described in the literature.
\end{abstract}

$T$ he ciliary body can give rise to congenital and acquired tumours. Congenital lesions are either choristomatous malformations or tumours composed of embryonic tissue. These include the rare glioneuroma and the less rare medulloepithelioma. The acquired lesions occur after embryonic development. Although some authors refer to the acquired tumours as adult diktyomas or adult medullopeitheliomas, the World Health Organisation prefers to diagnose these tumours as adenomas and carcinomas of the ciliary epithelium.

\section{CASE REPORT}

A 27 year old man presented with blurred vision of two years duration. There was no history of trauma, diabetes, or hypertension. Visual activity was reduced on the right and normal on the left. Intraocular pressure was normal. The right eye had a cataract. Gonioscopy showed a greyish white growth arising from the ciliary body, measuring approximately $1.5 \times 0.5 \mathrm{~cm}$, encroaching upon the lens and displacing it temporally. The angles were open and there was no hyperpigmentation. B scan revealed a growth arising from behind the iris from the ciliary body. The retina was intact. The patient underwent surgery, and sectoral cyclectomy with removal of the growth was performed.

\section{PATHOLOGICAL FINDINGS}

The ciliary body mass removed consisted of irregular, tan grey, non-pigmented tissue measuring $1.5 \times 0.5 \times 0.3 \mathrm{~cm}$. Microscopically, the tumour was arranged predominantly in a tubular and papillary configuration. The glandular lumina and intervening areas were composed of mucoid fibrillary material (fig 1). The cells lining these structures were round to polygonal, with round, bland nuclei and abundant eosinophilic cytoplasm. Mitoses were rare. Rare nuclei were enlarged. No significant inflammatory cell component was seen. Areas of calcification were present. The results of immunohistochemical studies were consistent with an origin from the non-pigmented ciliary epithelium (NPCE). The HMB45 antibody was negative, and the tumour stained positive for S100, vimentin, and cytokeratin (fig 2).

\section{DISCUSSION}

Primary tumours of the non-pigmented ciliary epithelium are rare. Zimmerman ${ }^{1}$ proposed dividing these tumours into two classes-congenital and acquired. Congenital tumours arise in the embryonic or early postnatal period, and include medulloepithelioma and glioneuromas. The acquired tumours are seen in adulthood and include Fuch's adenoma (hyperplasia of the NPCE, pseudoadenomatous hyperplasia, and coronal adenoma), ${ }^{1-3}$ adenoma, and adenocarcinoma of the non-pigmented ciliary epithelium.

Acquired tumours of the ciliary body have a wide spectrum of clinical and histopathological features and biological behaviour. Many are rare, and most studies have consisted of single case reports. ${ }^{4-6}$ Classification is based on the presence or absence of pigmentation, the cellular pattern, and malignant behaviour. Acquired neoplasms of the NPCE include Fuchs adenoma, adenoma, and carcinoma of the NPCE. ${ }^{15} 6$

\section{"Although benign, adenoma of the non-pigmented ciliary epithelium can behave aggressively locally, causing cataract and vitreous haemorrhage"}

Our patient developed a true adenoma of the NPCE, a benign tumour with variable histopathological features. The immunohistochemical results were consistent with an origin from the NPCE: S-100 and vimentin were positive. Immunohistochemistry for cytokeratin was also positivevariable staining for cytokeratin has been described in the literature. $^{7}$ Although benign, adenoma of the NPCE can behave aggressively locally, causing cataract and vitreous haemorrhage.

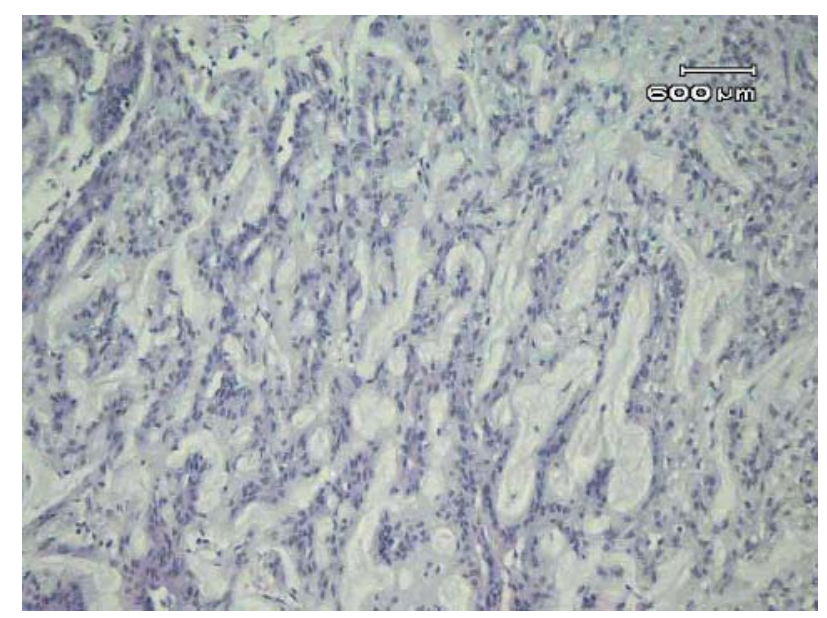

Figure 1 Photomicrograph of the tumour showing cords of nonpigmented epithelium separated by mucoid fibrillary matrix; haematoxylin and eosin stain.

Abbreviations: NPCE, non-pigmented ciliary epithelium 


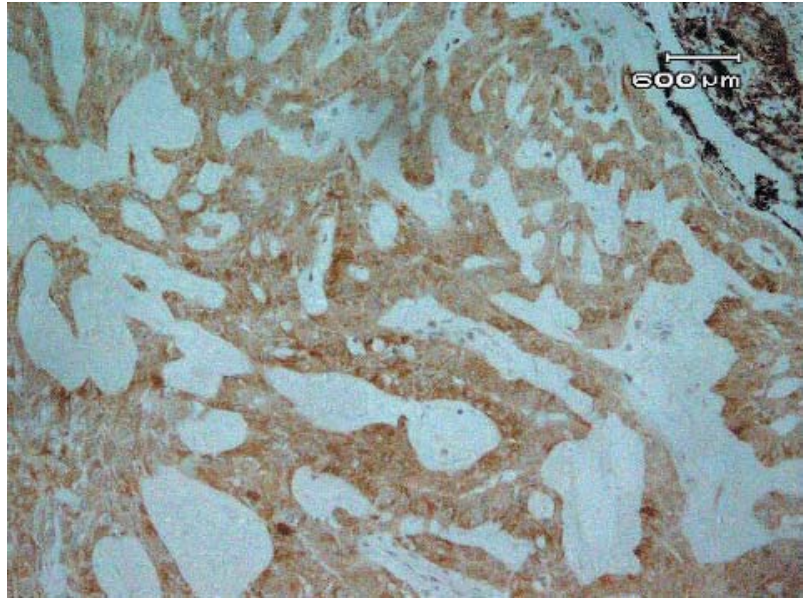

Figure 2 Photomicrograph of the tumour showing positive immunohistochemical staining for cytokeratin.

Adenomas are differentiated from adenocarcinoma of the NPCE by the absence of local infiltrative behaviour and a moderate degree of cellular pleomorphism with nuclear atypia. ${ }^{7}$ Rare mitoses are found. This tumour is seen in adulthood, with an age range of 24-70 years (mean, 45), and no sex predilection. The most common presenting symptom is visual loss. A ciliary body adenoma with smooth muscle differentiation has also been reported. ${ }^{8}$

Histopathological differential diagnosis of ciliary body adenoma includes ciliary body adenocarcinoma, adenoma of pigment epithelium, medulloepithelioma, in addition to lesions that occur in the deeper uveal tissue, such as melanoma, melanocytoma, metastatic carcinoma, leiomyoma, and neurilemoma.

\section{Authors' affiliations}

S Mansoor, A Qureshi, Department of Pathology, Shaukat Khanum Memorial Cancer Hospital and Research Centre, Johar Town, Lahore, Pakistan

\section{Take home messages}

- We report a case of ciliary body adenoma of the nonpigmented ciliary epithelium in a 27 year old Pakistani man-the first report of this entity in the Pakistani population to date

- The histological and immunohistochemical profiles were typical of the adenomas described in the literature-immunohistochemistry for S-100, vimentin, and cytokeratin was positive but the HMB45 antibody was negative

Correspondence to: Dr S Mansoor, Department of Pathology, Shaukat Khanum Memorial Cancer Hospital and Research Centre, Johar Town, Lahore, Pakistan; samina@skm.org.pk

Accepted for publication 8 April 2004

\section{REFERENCES}

1 Zimmerman LE. The remarkable polymorphism of tumors of the ciliary epithelium. Trans Aust Coll Ophthalmol 1970;2:114-25.

2 Shields JA, Shields CL. Tumors of the non-pigmented ciliary epithelium. In: Shields JA, Shields CL, eds. Intraocular tumors. A text and atlas. Philadelphia: WB Saunders, 1992:461-87.

3 Green WR, Retina. Acquired neuroepithelial tumors of the ciliary body. In: Spencer WH, Font RL, Homes EL, et al, eds. Ophthalmic pathology. An atlas and textbook, Vol. 2. Philadelphia: WB Saunders, 1985:1254-62.

4 Shields JA, Augsburger JJ, Waller PH, et al. Adenoma of the non pigmented epithelium of the ciliary body. Ophthalmology 1983;90:1528-30.

5 Grossniklaus HE, Lim Jl. Adenoma of the non-pigmented ciliary epithelium. Retina 1994;14:452-6.

6 McGowen HD, Simpsar ER, Hunter WS, et al. Adenoma of the non-pigmented ciliary epithelium. Can J Ophthalmol 1991;26:328-33.

7 Shields JA, Eagle RC. Acquired neoplasm of the non-pigmented ciliary epithelium (adenoma and adenocarcinoma). Ophthalomology 1996; 103:2007-16.

8 Shields JA, Eagle RC Jr, Shields CL. Adenoma of non-pigmented ciliary epithelium with smooth muscle differentiation. Arch Ophthalmol 1999;117:117-19. 